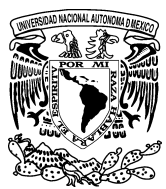

\title{
La participación laboral femenina y el uso del tiempo en el cuidado del hogar en México ${ }^{\text {is }}$
}

\author{
Women's labor participation and the use of time \\ in household care in Mexico
Armando Sánchez Vargas ${ }^{a}$, Ana Liz Herrera Merino ${ }^{b}$ e Ignacio Perrotini Hernández ${ }^{\text {b, } *}$

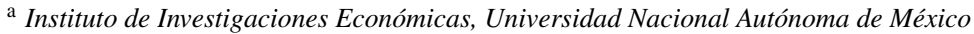

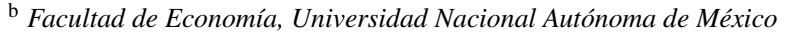 \\ Recibido el 2 de diciembre de 2014; aceptado el 15 de enero de 2015 \\ Disponible en Internet el 2 de julio de 2015
}

\begin{abstract}
Resumen
Este estudio ofrece evidencia empírica que sugiere que la participación femenina en el mercado laboral está condicionada por el uso de tiempo en el trabajo doméstico no remunerado en México. Esta situación se presenta en economías donde con frecuencia las mujeres son las que realizan las actividades relacionadas con la producción de bienes y servicios dentro del hogar y con el cuidado de sus integrantes. Las estimaciones se realizaron con información de la Encuesta Nacional de Uso del Tiempo de 2009 de los 32 estados de la República Mexicana. Se utilizó el modelo de selección de Heckman para corregir el sesgo de selección que puede existir en la muestra, ya que esta no fue diseñada para medir la situación laboral de las mujeres. Los resultados indican que las horas que las mujeres dedican al cuidado de niños y de personas mayores en el hogar, así como a la producción de bienes y servicios dentro del mismo, inciden negativamente en sus horas de trabajo remunerado.
\end{abstract}

\footnotetext{
is Este artículo es resultado directo de la investigación realizada a partir del apoyo financiero del proyecto PAPIIT «Participación femenina en el mercado laboral y el uso de tiempo en México: un análisis de pseudo panel» con clave IN301313-RN301313, así como resultado colateral del proyecto PAPIME-PE304914 y del proyecto SEDESOLCONACYT-2011-0167752.

* Autor para correspondencia.

Correo electrónico: iph@unam.mx (I. Perrotini Hernández).
}

La revisión por pares es responsabilidad de la Universidad Nacional Autónoma de México. 
Derechos Reservados ( 2015 Universidad Nacional Autónoma de México, Facultad de Contaduría y Administración. Este es un artículo de acceso abierto distribuido bajo los términos de la Licencia Creative Commons CC BY-NC-ND 4.0.

Palabras clave: Oferta laboral femenina; Cuidado del hogar; Trabajo doméstico; Uso de tiempo; Modelo de selección de Heckman

\begin{abstract}
This paper presents empirical evidence showing that women's time assigned to non-paid household work constrains their workforce supply in the formal labor market in México, where household duties and child and elderly care activities are mostly undertaken by women. Heckman's selection model is used in the paper to fix the potential selection bias in the sample as the latter does not directly measure female conditions in the labor market. Our results are based on data from the 2009 Time Use National Poll for the 32 states of the Mexican Republic.

All Rights Reserved (c) 2015 Universidad Nacional Autónoma de México, Facultad de Contaduría y Administración. This is an open access item distributed under the Creative Commons CC License BY-NC-ND 4.0 .
\end{abstract}

Keywords: Female labor supply; Household care; Household work; Time use; Heckman's selection model

Si Aristóteles hubiera guisado, mucho más hubiera escrito.

SOR JUANA INÉS DE LA CRUZ

\title{
Introducción
}

El estudio de la oferta laboral femenina es relevante debido a que tiene importantes implicaciones en diversos aspectos familiares, tales como el estado conyugal, la fertilidad, la distribución de los ingresos familiares y las brechas entre los salarios de hombres y mujeres (Killingsworth y Heckman, 1986).

Usualmente, la incorporación de las mujeres al mercado de trabajo se ha explicado a partir de factores individuales y de mercado. Sin embargo, existen pocos estudios que consideran el uso del tiempo en el hogar como uno de los principales determinantes de la oferta laboral femenina. En economías como la mexicana es posible que el tiempo que las mujeres dedican al trabajo doméstico ${ }^{1}$ explique la lenta incorporación de las mismas al mercado de trabajo, convirtiéndose así en una barrera de entrada.

El presente trabajo parte de la hipótesis de que el número de horas que una mujer destina al trabajo remunerado está condicionado por el tiempo que dedica a la producción de bienes y servicios dentro del hogar y al cuidado de los integrantes del mismo.

En este sentido, es importante desarrollar estudios que investiguen cuáles son los factores o barreras relacionados con el trabajo doméstico no remunerado que inciden directamente en la disponibilidad de tiempo de las mujeres para incorporarse a un trabajo remunerado, limitando así

\footnotetext{
${ }^{1}$ Actividades relacionadas con la preparación y servicio de alimentos para los integrantes del hogar, limpieza de la vivienda, limpieza y cuidado de ropa y calzado, mantenimiento, instalación y reparaciones a la vivienda y a los bienes de hogar, compras para los integrantes de hogar, pagos, trámites y administración del hogar y cuidado de personas que necesitan apoyo (menores de 15 años y mayores de 60).
} 
las oportunidades de progreso del género femenino en el ámbito laboral. Así, este tipo de análisis surge de la necesidad de realizar en México una adecuada planeación, diseño e implementación de políticas públicas cuyo objetivo principal sea reducir el tiempo que las mujeres emplean en dichos roles domésticos y ensanchar sus oportunidades de aumentar su oferta de horas de trabajo remunerado.

Específicamente, estudiamos el impacto que tiene en la oferta laboral femenina el tiempo invertido en el cuidado de niños y personas mayores, así como las horas que las mujeres ocupan para la realización del trabajo no pagado ${ }^{2}$. Esto permite verificar si la situación de las mujeres en el mercado de trabajo sigue siendo desventajosa con respecto a la de los hombres, dado que ellas no tendrían los mismos grados de libertad para elegir cuántas horas trabajar y cuántas horas dedicar a la producción de bienes y servicios en el hogar (Acosta, Perticara y Ramos, 2006) y al cuidado de su familia.

Las estimaciones de este estudio se hicieron a partir de la información de la Encuesta Nacional de Uso del Tiempo (ENUT) (INEGI, 2009); se considera como población objetivo a mujeres mayores de 12 años. Debido a que la muestra fue realizada sin considerar las condiciones de ocupación de las mujeres, es posible que exista un potencial sesgo de selección; para corregirlo se utilizó el modelo de selección de Heckman (1979

Los resultados del modelo revelan que las horas que una mujer destina a cuidar a niños menores de edad y a adultos mayores de 60 años son factores que inciden negativamente en sus horas de trabajo remunerado $(-0.0156$ y -0.014$)$. Asimismo, actividades como la preparación y el servicio de alimentos para los integrantes del hogar, limpieza de la vivienda, limpieza y cuidado de ropa y calzado, mantenimiento, instalación y reparaciones a la vivienda y a los bienes de hogar, compras para los integrantes del hogar, pagos, trámites y administración del hogar también tienen un impacto negativo en su disponibilidad de tiempo para incorporarse al mercado laboral $(-0.0121)$. Por otro lado, el salario es un factor que tiene un efecto positivo en la oferta laboral femenina (0.039). No obstante, la edad es un factor que afecta negativamente las horas de trabajo de una mujer (-0.0007). En conclusión, los resultados empíricos sugieren que el uso de tiempo de las mujeres en actividades domésticas tiene un papel relevante en la determinación y la disponibilidad de horas para trabajar. Dichas actividades pueden vincularse a los roles sociales y culturales que adoptan las mujeres dentro del hogar y que condicionan sus decisiones en el ámbito laboral y su participación en condiciones de igualdad en el mercado laboral.

El artículo está compuesto como sigue: la primera parte contiene una revisión bibliográfica de investigaciones previas sobre la oferta laboral femenina; en la segunda se desarrolla metodológicamente el modelo de selección de Heckman (1979); en la tercera parte presentamos algunos hechos estilizados de las horas de trabajo promedio de las mujeres en México y su relación con el uso de tiempo en actividades no remuneradas dentro del hogar; finalmente, en la cuarta parte se presentan los resultados del modelo y una discusión de estos corolarios.

\section{Revisión de la literatura}

La oferta laboral femenina es uno de los temas socioeconómicos que ha adquirido gran relevancia en los últimos años. Es por ello que se han realizado múltiples estudios que buscan explicar las marcadas diferencias entre la participación de la mujer y la del hombre en las actividades productivas y reproductivas (Gammage y Orozco, 2008). En el caso de México, una de estas

\footnotetext{
${ }^{2}$ No incluye el tiempo para el cuidado de personas que necesitan apoyo (menores de 15 años y mayores de 60 ).
} 
investigaciones es la que llevaron a cabo Arceo Gómez y Campos-Vázquez (2010) en la que estudian la oferta de fuerza de trabajo de las mujeres en México. Arceo y Campos concluyen que las horas trabajadas de las mujeres que tienen niños menores de 5 años son mucho más sensibles a los cambios de salarios en comparación con el caso de la mujer mexicana promedio, lo cual posiblemente se debe a que la distribución de su tiempo se limita por las responsabilidades que las mujeres tienen dentro del hogar.

Asimismo, en el estudio de Colinas (2008) se hace un diagnóstico de la relación que existe entre empleo, protección social y maternidad en el caso de México. El objetivo de dicho análisis era ofrecer un panorama general de la situación de las mujeres en el mercado laboral y su acceso a la protección social en México. Colinas (2008) concluye que en México las desigualdades — por ejemplo, la brecha salarial y la segregación ocupacional— persisten, situación que se explica por factores como la segregación en el mercado laboral y en la familia y por la asignación de tareas basadas en estereotipos de género.

Otra investigación que retoma las diferencias de género en el mercado laboral es la de Domínguez y Brown (2013), quienes encuentran que el mercado de trabajo no es neutro en cuestiones de género; la presencia de hijos y de adultos mayores tiene cierta influencia en las decisiones de las mujeres para trabajar en el domicilio, fuera del hogar o bien para permanecer al margen del mercado laboral. Domínguez y Brown (2013) proponen que el trabajo desde el domicilio es funcional por la presencia de hijos que dificulta la participación femenina fuera del hogar, lo que sugiere que el número de escuelas y guarderías con horarios adecuados para que las madres puedan tener más opciones laborales es insuficiente.

Licona (2000), por otra parte, analizó el efecto de la pobreza en el suministro de mano de obra femenina en el año 1992. El hallazgo principal de Licona (2000) consiste en que los salarios reales disminuyen cuando empeora la situación económica del país y que las mujeres que forman parte de familias de bajos ingresos aumentan su participación en el mercado laboral con la finalidad de mantener un nivel de consumo de subsistencia. Así, es frecuente que uno de los principales motivos por el cual las mujeres se incorporan al mercado laboral es para mantener un nivel de subsistencia o conservar un nivel de ingresos.La condición asimétrica adversa de la mujer en el mercado de trabajo es un fenómeno observable en otros países industrialmente rezagados, e incluso en países desarrollados. Por ejemplo, Ludin, Mörk y Öckert (2008) realizan estimaciones de los efectos que tiene la reducción de los costos del cuidado de los niños en la oferta laboral femenina en Estados Unidos, y concluyen que esa disminución depende del tipo de familia y de la región. Dasgupta y Goldar (2005), a su vez, encontraron resultados similares a los anteriores para las mujeres indígenas que están cerca de la línea de pobreza. Pencavel (1986) y Killingsworth y Heckman (1986) presentan análisis de la elasticidad salario de la oferta de trabajo para los hombres y las mujeres. Sus estimaciones indican que la elasticidad es muy cercana a cero para los hombres, mientras que para las mujeres resulta positiva y generalmente grande; aunque muy variable a través de diferentes especificaciones econométricas.

Uno de los estudios que evalúa las diferencias salariales de género en Georgia a partir del uso e implementación del modelo de selección de Heckman es el que realizó Khitarishvili (2009); sus resultados muestran que existe una diferencia sustancial de salarios por género en ese país. Además, sugiere que el difícil entorno económico social y las responsabilidades de cuidado que tienen las mujeres han generado una significativa discriminación femenina en el mercado laboral.

A pesar de los avances en el tema de equidad de género en el mercado laboral, aún quedan diferentes retos por superar y vacíos por colmar. A partir de la revisión de la literatura se advierte la existencia de pocos estudios que enfatizan el impacto del uso de tiempo en el trabajo doméstico sobre la oferta laboral femenina. Es decir, en qué medida las horas dedicadas al trabajo no 
remunerado y al cuidado de los integrantes del hogar reducen la disponibilidad de tiempo de las mujeres en México para ejercer su oferta de trabajo remunerado. La presente investigación contribuye a avanzar en el escrutinio de este tema hasta ahora insuficientemente estudiado. Con base en técnicas de corte transversal (Deaton, 1985) estudiamos la relación que existe entre, por un lado, la producción de bienes y servicios dentro del hogar y el cuidado de los integrantes del mismo y, por otro, la oferta laboral femenina en México.

\section{Metodología}

Dadas las características de la ENUT, cuya aleatoriedad no se realizó con base en la situación laboral de las personas, es posible que exista un potencial sesgo de selección. Por ello, decidimos que la metodología apropiada para este estudio es el modelo de selección de Heckman (1979) o modelo de 2 etapas. Este modelo econométrico nos permite determinar el impacto del tiempo dedicado a actividades del hogar y al cuidado de los integrantes de la familia en la oferta laboral femenina en México. El método consiste en 2 etapas: la primera etapa es un modelo tipo probit que estima la probabilidad de que, dadas ciertas características, una mujer decida trabajar o no trabajar en el sector formal. De esta estimación se obtiene el estadístico conocido como la razón inversa de Mills $\left(\hat{\lambda}=\hat{\phi}_{\mathrm{i}} / \hat{\Phi}_{\mathrm{i}}\right)$, que captura la magnitud del sesgo de selección. Posteriormente, la segunda etapa del modelo se estima a partir del método Mínimos Cuadrados Ordinarios (MCO), en la cual se incorpora la razón inversa de Mills como un regresor más del modelo. De esta forma, la significación del coeficiente $\lambda$ indica la magnitud de sesgo en que se incurriría si no se hubiese incorporado a la regresión explicativa, generando así coeficientes consistentes con el modelo MCO.

El modelo que explica las horas de trabajo de las mujeres se define como sigue:

$$
w_{i}=x_{i} \beta+Z_{i}+\varepsilon_{i}
$$

En donde: $w_{i}$ : horas de trabajo de la persona $i . x_{i}$ : vector de variables explicativas relacionadas con las horas de trabajo no remunerado de la persona $i$. $Z_{i}$ : vector de variables económicas y sociodemográficas de la persona $i$. $\varepsilon_{i}$ : variable del término de error. $w_{i}$ se observa únicamente para las mujeres que tienen un trabajo formal.

Por otro lado, la ecuación de selección se formula de la siguiente manera:

$$
g_{i}=\delta+m_{i} \gamma+u_{i}
$$

En donde: $g_{i}$ : toma el valor de 1 si la persona trabaja en el sector formal, y de 0 en otro caso. $m_{i}$ : vector de variables que podrían explicar que una mujer decida trabajar en el sector formal o fuera de este. $u_{i}$ : variable del término de error.

A partir de la razón inversa de Mills se puede obtener evidencia de la existencia del sesgo de selección.

Por lo tanto, si $g_{i}=1$, la ecuación resultante del modelo es:

$$
w^{*}=\beta_{0}+\beta x+\varepsilon
$$

Y la ecuación de participación es:

$$
g=1\left(\gamma^{\prime} z+\mathrm{v}\right)
$$


Tabla 1

Horas de trabajo remunerado e ingreso laboral por género

\begin{tabular}{llr}
\hline & Horas trabajadas & Ingreso $^{\text {a }}$ \\
\hline Mujeres & 193.19 & 4,600 \\
Hombres & 252.33 & 5,000 \\
\hline
\end{tabular}

Fuente: elaboración propia con datos de la Encuesta Nacional de Uso del Tiempo 2009.

a Debido a la distribución de la variable de ingreso, se estimó la mediana.

$$
\left[\begin{array}{l}
\mathrm{u} \\
\mathrm{v}
\end{array}\right] \sim N\left(\left[\begin{array}{l}
0 \\
0
\end{array}\right],\left[\begin{array}{ll}
\sigma_{\mathrm{u}}^{2} & \rho \\
\rho & 1
\end{array}\right]\right)
$$

Del modelo Tobit se sabe que:

$$
E=[w \mid x, z, s=1]=x \beta+\rho \lambda(z \gamma)
$$

Donde $\lambda$ () es la inversa de la razón Mills y está correlacionada con $\varepsilon$.

\section{Hechos estilizados}

Con el objetivo de hacer un análisis estadístico de las horas que las mujeres dedican al mercado laboral y del nivel de ingresos que obtienen, especificamos algunos hechos estilizados con datos de la Encuesta Nacional sobre el Uso del Tiempo (INEGI, 2009). Esa encuesta contiene información a nivel nacional del tiempo que las personas destinan a sus actividades diarias. Esto proporcionó los insumos de información necesarios para la medición de variables relacionadas con el trabajo remunerado y el no remunerado de las mujeres mexicanas. El análisis estadístico se realizó únicamente para mujeres con las siguientes características: a) tienen un empleo; b) perciben ingresos por concepto de su trabajo, y c) reportan un número de horas laborales mayor a cero. Una de las características de la definición de la Población Económicamente Activa (PEA) del Instituto Nacional de Estadística y Geografía (INEGI) es que la persona debe tener al menos 12 años de edad. Para fines del estudio, es importante considerar únicamente a las personas que reportan un nivel de ingresos así como un número positivo de horas de trabajo.

La diferencia de género es uno de los aspectos de mayor relevancia en el estudio del mercado de trabajo. La tabla 1 muestra que en promedio existe una gran diferencia entre las horas de trabajo que mensualmente realizan las mujeres y las que realizan los hombres; los datos revelan que existe una brecha de 59.14 horas entre ambos géneros. En el caso de las mujeres, esta situación podría explicarse por factores relacionados con el cuidado del hogar y de los integrantes del mismo. También se puede ver una desigualdad en el nivel de ingreso laboral de las mujeres y el de los hombres. Debido a que la distribución de los ingresos tiene un sesgo, calculamos la mediana, y con base en ello se observa que en promedio los hombres ganan mensualmente 400 pesos más que las mujeres.

Así, dentro de los hogares existen características que podrían determinar la oferta laboral femenina. Por ejemplo, aspectos relacionados con la estructura del hogar, como la presencia de niños y adultos mayores, podrían representar un consumo de tiempo para las mujeres y generar así un aumento de sus horas de trabajo no remunerado y una disminución del tiempo de trabajo remunerado. A partir de los datos de la tabla 2 se puede distinguir una relación entre la presencia de algunos integrantes del hogar que requieren cuidados y las horas de trabajo de las mujeres. 
Tabla 2

Horas de trabajo formal remunerado e ingreso laboral de mujeres empleadas condicionadas por la estructura del hogar

\begin{tabular}{lcc}
\hline & Horas trabajadas & Ingreso $^{\text {a }}$ \\
\hline Con niños menores de 6 años en el hogar & 179.40 & 4,000 \\
Sin niños menores de 6 años en el hogar & 198.69 & 4,800 \\
Con niños de 6 a 14 años en el hogar & 183.59 & 4,000 \\
Sin niños de 6 a 14 años en el hogar & 200.43 & 5,000 \\
Con personas mayores a 60 años en el hogar & 199.99 & 4,600 \\
Sin personas mayores a 60 años en el hogar & 191.90 & 4,700 \\
Con ayuda de otra mujer en el hogar & 202.26 & 4,500 \\
Sin ayuda de otra mujer en el hogar & 177.88 & 5,000 \\
Con presencia de un adulto varón en el hogar & 192.15 & 4,600 \\
Sin presencia de un adulto varón en el hogar & 197.94 & 5,000 \\
\hline
\end{tabular}

Fuente: elaboración propia con datos de la Encuesta Nacional de Uso del Tiempo 2009.

a Debido a la distribución de la variable de ingreso, se estimó la mediana.

Es decir, la tabla 2 muestra las horas de trabajo de las mujeres condicionadas por la presencia o ausencia de: a) niños menores de 15 años; b) personas mayores, y c) mujeres y hombres adultos.

Uno de los aspectos más relevantes que muestra la tabla 2 es que cuando hay al menos otra mujer en el hogar la oferta laboral remunerada femenina es mayor que cuando solo hay una mujer: 202.26 y 177.88 horas mensuales, respectivamente (Sánchez Vargas, Villarespe, Román y Herrera (2014). En consecuencia, se puede decir que posiblemente la presencia de otra mujer en el hogar podría representar una ayuda en las actividades domésticas. Por el contrario, el tiempo de trabajo es inferior cuando hay un adulto varón en el hogar (192.15 horas), así como cuando hay niños menores de 15 años (183.59 horas). La información de la misma tabla sugiere que cuando hay adultos mayores de 60 años en el hogar el promedio de horas de trabajo aumenta. Lo anterior puede explicarse porque en ocasiones los adultos mayores también contribuyen a las actividades domésticas y al cuidado de otros miembros de la familia. Los datos también muestran que conforme aumenta el número de horas de trabajo, es posible que los niveles de ingreso también sean mayores.

Con la finalidad de enriquecer este análisis, la tabla 3 ofrece información del promedio de horas que una mujer trabaja cuando también dedica tiempo al apoyo y cuidado de los integrantes del hogar, tales como niños y personas mayores. En promedio, las mujeres que destinan al menos

Tabla 3

Horas de trabajo formal remunerado e ingreso laboral de mujeres empleadas condicionadas por el cuidado de los miembros del hogar

\begin{tabular}{llc}
\hline & Horas trabajadas & Ingreso $^{\text {a }}$ \\
\hline Dedica tiempo a cuidar niños menores de 6 años & 170.77 & 4,200 \\
No dedica tiempo a cuidar niños menores de 6 años & 199.64 & 4,700 \\
Dedica tiempo a cuidar niños menores de 15 años & 166.70 & 4,500 \\
No dedica tiempo a cuidar niños menores de 15 años & 210.54 & 4,800 \\
Dedica tiempo a cuidar personas mayores de 60 años & 179.49 & 4,200 \\
No dedica tiempo a cuidar personas mayores de 60 años & 193.74 & 4,800 \\
\hline
\end{tabular}

Fuente: elaboración propia con datos de la Encuesta Nacional de Uso del Tiempo 2009.

a Debido a la distribución de la variable de ingreso, se estimó la mediana. 
Tabla 4

Horas de trabajo formal remunerado e ingreso laboral de mujeres empleadas condicionadas por trabajo doméstico no remunerado

\begin{tabular}{llc}
\hline & Horas trabajadas & Ingreso $^{\mathrm{a}}$ \\
\hline Con horas de trabajo no pagado & 191.74 & 4,600 \\
Sin horas de trabajo no pagado & 272.90 & 5,600 \\
Realiza trabajo doméstico no pagado & 191.84 & 4,600 \\
No realiza trabajo doméstico no pagado & 284.63 & 6,000 \\
\hline
\end{tabular}

Fuente: elaboración propia con datos de la Encuesta Nacional de Uso del Tiempo 2009.

a Debido a la distribución de la variable de ingreso, se estimó la mediana.

una hora al cuidado de niños menores de 6 años de edad trabajan menos tiempo respecto de las que no tienen que cuidar infantes. Estos hechos estilizados muestran que el tiempo que demandan los niños pequeños podría incidir en la oferta laboral femenina, dado que si las mujeres dedican tiempo al cuidado infantil trabajan en promedio 170.77 horas al mes, y si no consagran tiempo a ese cuidado, trabajan 199.64 horas en el mercado laboral remunerado. Lo anterior muestra que existe una diferencia de 28.87 horas entre ambos grupos de mujeres. Además, en estas circunstancias también hay discrepancias en su nivel de ingresos mensual, lo que puede sugerir la existencia de una probable asociación entre el nivel de ingresos y las horas trabajadas.

Asimismo, cuando las mujeres dedican tiempo al cuidado de niños menores de 15 años el promedio de su tiempo de trabajo remunerado es mucho menor en comparación con aquellas que no destinan tiempo al cuidado de niños. Una mujer que cuida niños menores de 15 años destina únicamente 166.70 horas al trabajo remunerado; en cambio, cuando no invierte tiempo en el cuidado de niños menores su tiempo de trabajo aumenta a 210.54 horas mensualmente. Todo esto sugiere que el cuidado de niños menores de edad podría condicionar la oferta laboral femenina, incidiendo de modo adverso al mismo tiempo en su nivel de ingresos.

Por otro lado, el cuidado de las personas mayores de 60 años dentro del hogar también puede demandar un tiempo significativo y puede incidir en la disponibilidad de tiempo y de oferta de trabajo remunerado de las mujeres. De acuerdo con a la tabla 3, las mujeres que brindan apoyo a las personas mayores reportan un menor número de horas de trabajo vis-à-vis aquellas que no asisten a personas mayores: 179.49 y 193.74 horas, respectivamente. Todo lo anterior hace suponer que el tiempo de trabajo que las mujeres dedican al cuidado de los miembros del hogar podría representar una restricción para que ellas incrementen su tiempo de trabajo remunerado y/o, en el escenario más drástico, un óbice para su incorporación en el mercado laboral formal. En consecuencia, es posible que esta condición restrictiva limite la posibilidad de que las mujeres perciban un nivel de ingresos superior que les permitiría garantizar una calidad de vida mejor.

Otra de las actividades que se relaciona directamente con el trabajo doméstico que realizan las mujeres dentro del hogar es la producción de bienes y servicios. Es decir, actividades domésticas como la preparación y servicio de alimentos para los integrantes del hogar, limpieza de la vivienda, limpieza y cuidado de ropa y calzado, mantenimiento, instalación y reparaciones de la vivienda y de los bienes de hogar, compras para los integrantes del hogar, pagos, trámites y administración del hogar.

Los datos de la tabla 4 revelan que las mujeres que dedican tiempo a este tipo de actividades no remuneradas en promedio tienen menos horas de trabajo remunerado que aquellas que no realizan actividades del hogar no remuneradas: 191.74 horas en el caso de las primeras y 272.90 horas en el de las segundas. Esto revela que existe una diferencia de 81.16 horas entre las mujeres que reportan 
que realizan trabajo del hogar no pagado y las que no lo realizan ${ }^{3}$. Estos hechos estilizados hacen alusión a que el trabajo no pagado es uno de los factores que determina la oferta laboral de las mujeres.

El trabajo doméstico es, de facto, parte esencial del trabajo no remunerado que las mujeres realizan en los hogares (Pedrero, 2010). El trabajo doméstico involucra las actividades relacionadas con la producción de bienes y servicios dentro del hogar, así como el cuidado de los integrantes del mismo. Por esta razón, también es importante hacer el análisis de las horas de trabajo condicionadas al tiempo que las mujeres dedican a la realización de labores domésticas. Los datos revelan empíricamente que el trabajo doméstico no remunerado podría influir negativamente en la oferta de trabajo remunerado de las mujeres. En promedio, el tiempo de trabajo de una mujer es menor cuando realiza actividades no remuneradas (191.84 horas). Por otro lado, cuando las mujeres no destinan tiempo a las labores no remuneradas del hogar su tiempo de trabajo es mayor (284.63 horas). Lo anterior revela que la brecha de tiempo de trabajo entre las mujeres que realizan trabajo doméstico y las que no lo realizan es de 92.79 horas.

\section{Resultados del modelo}

Las estimaciones que sustentan la hipótesis de este estudio se realizaron a partir del modelo de selección de Heckman (1979), dado que es posible que exista un potencial sesgo de selección en la información de la ENUT que no considera la situación laboral de los individuos. Con esta técnica, estimamos 2 modelos simultáneamente. El primer modelo es el de selección, el cual muestra los factores que determinan la probabilidad de que una mujer decida o no trabajar en el sector formal. La segunda etapa muestra el efecto que tienen las variables de uso de tiempo en tareas del hogar de las mujeres en sus horas de trabajo remunerado e incorpora como coeficiente la razón inversa de Mills. Es decir, este modelo muestra el impacto de las horas de trabajo no remunerado de las mujeres en su disponibilidad para incorporarse al mercado de trabajo remunerado.

La tabla 5 muestra los resultados del modelo de selección y del modelo de las horas de trabajo femenino. Los factores que se incorporan como determinantes en la ecuación de las horas de trabajo son: a) horas para el apoyo y el cuidado de menores de 6 años, menores de 15 años y personas mayores; b) horas dedicadas al trabajo no pagado en casa, y c) la edad.

Los resultados de la primera etapa del modelo sugieren que el factor que tiene un mayor impacto positivo en la probabilidad de que una mujer tenga un trabajo formal remunerado es el ingreso (0.434). Además, el nivel de educación es otro determinante que también aumenta esa probabilidad para las mujeres (0.1566). Esto sugiere que cuando una mujer tiene un mayor grado de escolaridad sus posibilidades de incorporarse al sector formal remunerado aumentan, y esto se explica porque un mayor nivel educativo otorga una mayor probabilidad de encontrar un empleo pagado en el sector formal. Por otro lado, la edad tiene un efecto negativo en la incorporación de las mujeres al mercado de trabajo formal (-0.0007); a mayor edad, más difícil resultará su integración en el mercado de trabajo. Otro aspecto que es relevante y que influye de forma negativa en las probabilidades de que una mujer tenga un trabajo formal son las horas que dedica al trabajo no pagado en casa. Específicamente, actividades relacionadas con la preparación y servicio de alimentos para los integrantes del hogar, limpieza de la vivienda, limpieza y cuidado de ropa y calzado, mantenimiento, instalación y reparaciones a la vivienda y a los bienes del hogar, compras para los integrantes del hogar, pagos, trámites y administración del hogar (-0.0099).

\footnotetext{
${ }^{3}$ Se realizó una prueba de hipótesis de diferencia de medias que fue estadísticamente significativa $[\operatorname{Pr}=0.000]$.
} 
Tabla 5

Resultados del modelo

\begin{tabular}{lc}
\hline Variable dependiente: horas trabajadas & Coeficiente \\
\hline Ingreso & $0.039[0.0108]$ \\
Apoyo a menores de 6 años & $-0.0054[0.0014]$ \\
Apoyo a menores de 15 años & $-0.0156[0.0006]$ \\
Apoyo a personas mayores de 60 años & $-0.014[0.0021]$ \\
Trabajo no pagado en casa ${ }^{a}$ & $-0.0121[0.0005]$ \\
Edad & $0.026[0.0037]$ \\
Edad2 & $-0.0003[0.0000]$ \\
Constante & $4.574[0.1244]$ \\
Modelo de selección: Trabajo formal =1 & \\
Ingreso & $0.4343[0.0198]$ \\
Educación & $0.1566[0.0087]$ \\
Edad & $0.0561[0.0077]$ \\
Edad2 & $-0.0007[0.0000]$ \\
Trabajo no pagado en casa ${ }^{a}$ & $-0.0099[0.0012]$ \\
Constante & $-5.2662[0.1869]$ \\
Rho & 0.4252 \\
Sigma & 0.3463 \\
Lambda & 0.1472
\end{tabular}

Todos los resultados tienen el $99 \%$ de confianza.

Número de observaciones: 7,706; observaciones censuradas: 5,027; observaciones no censuradas: 2,679.

Arthrho P> $|\mathrm{z}|: 0.000$.

Fuente: elaboración propia con base en la estimación de modelos con información de la Encuesta Nacional de Uso del Tiempo 2009.

a Actividades relacionadas con la preparación y servicio de alimentos para los integrantes del hogar, limpieza de la vivienda, limpieza y cuidado de ropa y calzado, mantenimiento, instalación y reparaciones a la vivienda y a los bienes de hogar, compras para los integrantes de hogar, pagos, trámites y administración del hogar.

En la segunda etapa del modelo se estimaron los determinantes de las horas de trabajo de las mujeres en México. Los resultados revelan que factores económicos como el ingreso inciden positivamente en la oferta laboral de las mujeres (0.039). Lo cual quiere decir que un aumento en el salario incentiva a las mujeres para trabajar un mayor número de horas. No obstante, existen otros factores que se asocian al cuidado de los integrantes del hogar y a la producción de bienes y servicios dentro del mismo que también impactan el número de horas de trabajo remunerado de las mujeres. Los resultados revelan que cuando las mujeres dedican tiempo al cuidado de los integrantes más vulnerables del hogar su disponibilidad para trabajar en el mercado laboral remunerado disminuye.

Específicamente, la información de la tabla 5 ofrece evidencia que las horas que las mujeres destinan al apoyo y cuidado de niños menores de 6 años afectan negativamente sus horas dedicadas a un trabajo remunerado ( -0.0054$)$. Sin embargo, el impacto negativo es mayor cuando una mujer dedica horas de cuidado a niños menores de 15 años (-0.0156). Asimismo, las horas de apoyo a un adulto mayor también tienen un efecto negativo en las horas de trabajo remunerado de las mujeres (-0.014). Lo cual significa que el tiempo que demandan algunos integrantes del hogar (niños y personas mayores) reduce el tiempo que una mujer puede laborar en el mercado de trabajo pagado.

Otro aspecto que también limita la oferta laboral de las mujeres es la cantidad de tiempo que dedican a la producción de bienes y servicios para el hogar, dando así origen al trabajo no pagado. Con base en estos resultados, se puede sostener que las horas de trabajo no pagado influyen 
negativamente en el trabajo remunerado de las mujeres $(-0.0121)$. Lo anterior sugiere que la demanda de tiempo del cuidado de los hogares y de sus integrantes es un elemento que reduce la disponibilidad de tiempo de las mujeres para incorporarse al mercado de trabajo en condiciones económicas al menos similares al de las mujeres (y hombres) que no están confinados a distribuir su tiempo de trabajo entre actividades no remuneradas y actividades pagadas. Por otra parte, es importante mencionar que la edad de una mujer incide negativamente en su tiempo de trabajo en el mercado laboral remunerado, dado que conforme aumenta la edad, su disponibilidad de tiempo para trabajar en él disminuye $(-0.0003)$.

A partir de estas estimaciones se puede concluir que las mujeres no solo deben elegir cuántas horas dedicar a un trabajo remunerado, sino también cuanto tiempo destinar al trabajo doméstico no remunerado. De esta manera, los datos confirman la vigencia social de la inequidad de género en el mercado de trabajo. Por otro lado, es importante mencionar que, de acuerdo con el signo positivo del coeficiente de la razón inversa de Mills (Rho) del modelo, es posible afirmar que los residuales de cada uno de los modelos están correlacionados positivamente, lo cual quiere decir que factores no observables del modelo de selección afectan positivamente la oferta de horas de trabajo (0.4252).

\section{Conclusiones}

La oferta laboral femenina no solo depende de aspectos económicos, sino también de factores sociales (y aun culturales) relacionados con el uso del tiempo dentro del hogar. Es posible que lo anterior se asocie a cuestiones sociales y culturales que se han relacionado directamente con el quehacer tradicional (no natural) de las mujeres dentro del hogar.

El objetivo principal de este estudio consiste en ofrecer evidencia de que la disponibilidad del tiempo de las mujeres para incorporarse a un trabajo formal remunerado también podría estar sujeta al uso de tiempo en actividades dentro del hogar. Los resultados muestran que el tiempo que demanda la producción de bienes y servicios dentro del hogar, así como el cuidado de los integrantes del mismo, influyen en el número de horas de trabajo remunerado de las mujeres, limitando de esta manera las oportunidades de progreso del género femenino en el ámbito laboral.

A partir del modelo de selección de Heckman (1979) encontramos que el tiempo que las mujeres dedican al cuidado de niños menores de 6 y 15 años y de personas mayores de 60 años incide negativamente en su disponibilidad de horas para un trabajo remunerado. Esto se debe a que estos miembros del núcleo familiar requieren más atención y cuidados que normalmente brindan las mujeres. Aunado a lo anterior, las estimaciones también ofrecen evidencia de que las horas de trabajo no pagado, que por tradición desempeñan las mujeres (preparación de alimentos, limpieza de la vivienda, mantenimiento de la vivienda, compras, pagos y administración del hogar), también reducen sus posibilidades de incrementar su oferta de trabajo remunerado, limitando así su incorporación al mercado de trabajo formal.

Con base en los resultados de este estudio inferimos que las actividades relacionadas con el uso del tiempo de la mujer dentro de los hogares podrían limitar las condiciones en que las mujeres en general pueden incorporarse al mercado laboral. De esta forma también se reducen sus posibilidades de percibir los ingresos suficientes para una subsistencia adecuada de ellas y de sus familias, particularmente cuando la mujer es la proveedora (única o principal) del hogar.

Una primera recomendación que emerge de este estudio es la pertinencia de diseñar y desarrollar políticas públicas orientadas a proporcionar la ayuda necesaria dentro de los hogares mediante acciones que tengan como principal objetivo liberar el tiempo de trabajo de las mujeres dedicado a las actividades domésticas. Por ejemplo, impulsar programas ya existentes como las 
guarderías públicas y escuelas de tiempo completo que permitan a las mujeres tener una mayor flexibilidad en su uso del tiempo y así poder ampliar sus posibilidades de incorporación al mercado laboral.

Segundo, la importancia de desarrollar este tipo de políticas públicas surge de la necesidad de promover al mismo tiempo la equidad de género dentro del mercado de trabajo, ofrecer mayores posibilidades a las mujeres mexicanas para integrarse al trabajo formal y mejorar su calidad de vida. Puesto que una sociedad con equidad de género en el trabajo es, al mismo tiempo, una sociedad con índices de civilidad y de participación democrática más edificantes.

\section{Referencias}

Acosta, E., Perticara, M. y Ramos, C. (2006). Empleo femenino: Oferta laboral y cuidado infantil. Washington D.C: Banco Interamericano de Desarrollo.

Arceo Gómez EO, Campos-Vázquez RM (2010). Labor Supply of Married Women in Mexico: 1990-2000. Documento de Trabajo, Centro de Estudios Económicos, Colegio de México (XVI): 1-35.

Colinas L (2008). Economía productiva y reproductiva en México: un llamado a la Conciliación. Serie Estudios y Perspectivas, No. 94, CEPAL/México.

Dasgupta, P. y Goldar, B. (2005). Female labour supply in rural India: An econometric analysis. India: Institute of Economic Growth, Delhi University Enclave.

Deaton, A. (1985). Panel data from a time series of cross-sections. Journal of Econometrics, 30, 109-126.

Domínguez, L. y Brown, F. (2013). Diferencias de género en la elección del sitio de trabajo en un contexto de crisis. CEPAL, 111, 83-102.

Gammage S, Orozco M (2008). El trabajo productivo no remunerado dentro del hogar: Guatemala y México. Serie Estudios y Perspectivas, No. 103, CEPAL/México.

Heckman, J. (1979). Sample selection bias as a specification error. Econometrica, 47, 153-162.

Instituto Nacional de Estadística, Geografía e Informática (INEGI) (2009). Encuesta Nacional sobre el Uso del Tiempo (ENUT).

Killingsworth, M. R. y Heckman, J. J. (1986). Female labor supply: A survey. Handbook of Labor Economics, 1, $103-204$.

Khitarishvili T (2009). Explaining the gender wage gap in Georgia. The Levy Economics Institute, Working Paper No. 577.

Licona, G. (2000). Reshaping the labor supply curve for the poor. Río de Janeiro, Brasil: Annual Meeting of the Latin American and Caribbean Economic Association.

Ludin, D. E., Mörk, E. y Öckert, B. (2008). How far can reduced childcare prices female labor supply? Labor Economics, 14, 647-659.

Pedrero M (2010). Valor económico del trabajo doméstico en México. Aportaciones de mujeres y hombres. México: INMUJERES [consultado 3 Mar 2014]. Disponible en: http://www.inmujeres.gob.mx/images/stories/cuadernos/ ct20.pdf

Pencavel, J. (1986). Labor supply of men: A survey. En O. Ashenfelter y D. Card (Eds.), Handbook of Labor Economics (Vol. 1) (pp. 3-102). Elsevier Science Publishers.

Sánchez Vargas A, Villarespe RV, Román CD, Herrera MA (2014). La participación laboral femenina y la estructura de los hogares en México: un enfoque de pseudo panel (2005-2010). Artículo en dictamen en la Revista CEPAL. 